\title{
A Rare Case of Aortico-Right Ventricular Tunnel Along with Ventricular Septal Defect Presented as Ruptured Sinus of Valsalva in an Adult Female
}

\author{
Binay Krishna Sarkar*, Krishnendu Chakraborty, Dilip Saha, Paresh Banerjee \\ Department of Cardiovascular \& Thoracic Surgery, Nil Ratan Sircar Medical College \& Hospital, Kolkata, India \\ Email: "binayskr@hotmail.com
}

Received April 8, 2013; revised May 13, 2013; accepted May 23, 2013

Copyright (C) 2013 Binay Krishna Sarkar et al. This is an open access article distributed under the Creative Commons Attribution License, which permits unrestricted use, distribution, and reproduction in any medium, provided the original work is properly cited.

\begin{abstract}
Background: Aortico-right ventricular tunnel is an extremely rare congenital defect rarely described in an infant. This diagnosis is likely to be missed due to its rare entity and similar clinical presentations with other aortico-right ventricular communications like ruptured sinus of valsalva. Methods: We report a case of previously undiagnosed aortico-right ventricular tunnel along with a perimembraneous ventricular septal defect in an 18-year-old female. She had history of exertional dyspnoea, palpitation, history of recurrent lower respiratory tract infection. She was diagnosed as a case of ruptured sinus of valsalva (RSOV) elsewhere. She had "to and fro" murmur, features of congestive cardiac failure. Her echo diagnosis was RSOV. On surgical exploration, after opening the aorta, we found a tunnel like opening in the aorta leading to the roof of RV cavity in between right and non coronary sinuses at the commissural level. Cusps were splayed wide part. Ventricular septal defect was conspicuous from right atrial approach. Post operative CT angio was done. Results: Venricular septal defect was closed from the right atrial approach and aortico-right ventricular tunnel was repaired through aortic and right venricular approach. Postoperative CT angio also confirmed the location and closure of the defects. Postoperative recovery was uneventful. Conclusions: Aortico-right ventricular tunnel in an adult female has not been reported in the literature previously. This rare entity should be considered in the differential diagnosis of a critically ill patient with a "to and fro" murmur, and signs of right heart failure.
\end{abstract}

Keywords: Aortico-RV Tunnel; Ventricular Septal Defect

\section{Introduction}

Aneurysm of the sinus of valsalva is a rare aortic lesion $[1,2]$. Congenital aneurysms are caused by weakness at the junction of the aortic media and the annulus fibrosus. Congenital aneurysms are more common than acquired aneurysms. Acquired lesions occur secondary to disease processes that involve the aortic root like syphilis and infective endocarditis [1,2]. It can arise from the right coronary $(77 \%)$, noncoronary $(23 \%)$ and, sometimes, the left coronary sinus of valsalva [2]. It can remain asymptomatic for many years. About half are associated with aortic regurgitation. Symptoms can be caused by mechanical obstruction, with compression of the conducting system, or intracardiac rupture. Rupture of sinus of valsalva aneurysms may be spontaneous, or triggered by chest trauma or exertion, or iatrogenic (cardiac catheterization). Rupture can occur into the right ventricle, right

*Corresponding author. atrium or left atrium and rarely into the left ventricle or interventricular septum $[1,2]$. A ruptured aneurysm of the sinus of valsalva can present with acute congestive heart failure and can be life threatening. Once an aneurysm rupture, the median survival is reduced to one to two years (if left untreated) and patients usually succumb to cardiac failure or infective endocarditis [2]. Prompt surgical repair is the optimal therapy after the diagnosis is made. The concomitant surgical repair of associated ventricular septal defect, atrial septal defect, and the aortic valve is often required.

Although ruptured sinus of valsalva is a rare but well-known congenital anomaly, aortico-right ventricular tunnel is an exceptionally rare entity [3-7]. Aortico-right ventricular tunnel is an extremely rare congenital defect rarely described in the literature especially in infants [3-8]. This diagnosis is likely to be missed due to its rare entity and similar clinical presentations with other aortico-right ventricular communications like ruptured sinus 
of valsalva. We report a female patient with aortico-right ventricular tunnel who was seen in adulthood and was successfully treated by surgical closure.

Case Summary: An 18-year-old female was admitted in the department of Cardiothoracic and vascular surgery with the history of exertional dyspnoea, palpitation, history of recurrent lower respiratory tract infection. She was diagnosed as a case of ruptured sinus of valsalva (RSOV) elsewhere. She had also history of hospitalization for heart failure. She had NYHA (New York Heart Association) class 3 dyspnoea on admission and was on multiple cardiac medications including antifailure treatment. She had no history of syncopal attacks, chest pain or hemoptysis. She had no history of diabetes. On examination, she was found to have features of cardiomegaly, hepatomegaly, bilateral crepitations in chest, with continuous murmur in the left parasternal area best heard over the $3^{\text {rd }}$ intercostal space. Her electocardiogram revealed left ventricular hypertrophy. Echo doppler study revealed left to right shunt in the region of the right aortic sinus and another shunt in the ventricular septum in the perimembraneous area along with aortic regurgitation.

Surgery: On surgical exploration, after opening the aorta, we found a tunnel like opening in the aorta (Figure 1) leading to the roof of right ventricular (RV) cavity in between right and non coronary sinuses at the commissural level. Cusps were splayed wide part. Right atrium was opened via an incision parallal to the atrio-ventricular groove and ventricular septal defect was delineated through tricuspid valve. Right ventricle was opened and

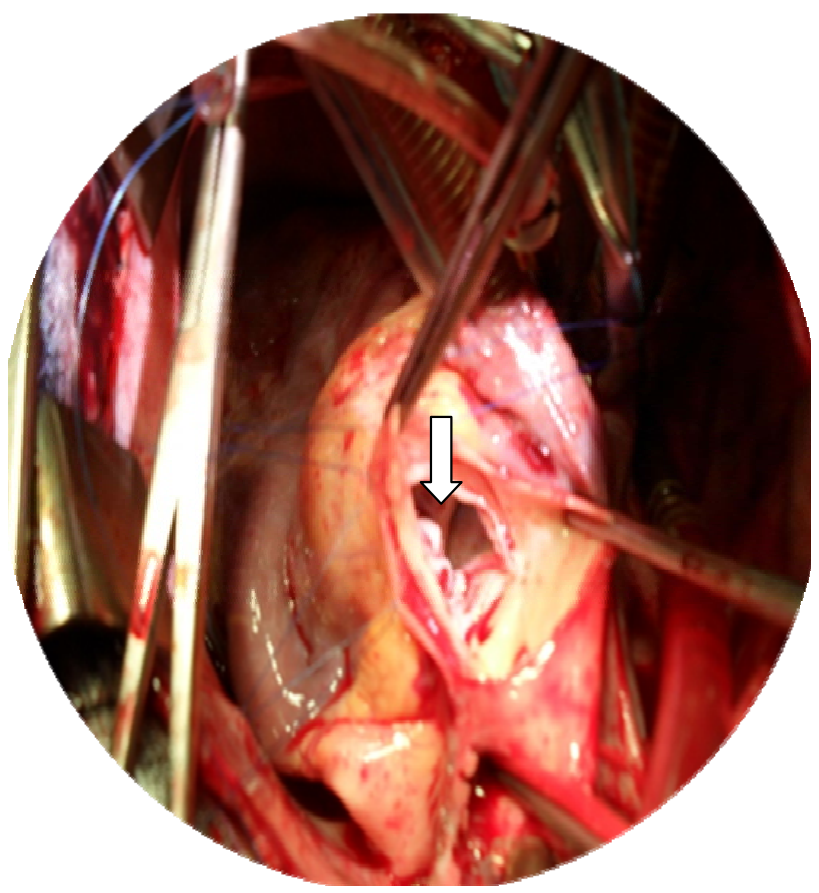

Figure 1. Peroperative picture showing aortic end of the aortic $R V$ tunnel. the aortico-right ventricular tunnel was confirmed through aorta and right ventricle and closed with a similar autologous treated pericardial patch.

Ventricular septal defect was conspicuous from right atrial approach which was confirmed as a separate defect and it was repaired with autologous treated pericardial patch. Aortic valve cusps, aortic annulus and coronaries were normal. Aortic valve repair was done without distorting valve cusps. Post-operative computed tomogram (CT) angiography was done.

This rare diagnosis could be documented postoperatively by Computed Tomography angio study and three dimensional reconstructions which clearly showed the location of the tunnel (Figure 2) with two separate defects which were repaired with patch (Figures 3(a) and (b)). Sinus of valsalva was found normal and sinus of valsalva aneurysm was excluded. Location of the Aorticoright ventricular tunnel defect was clearly shown between the right coronary and non coronary sinuses adjacent to the roof of the right ventricle.

Postoperative recovery had been uneventful. There was mild elevation of serum bilirubin in the immediate postoperative period which became normal within few days. She was discharged after ten days without any infective complication. She was doing well during her follow-up visits.

\section{Discussion}

Aortico-right ventricular tunnel is a rare cardiac entity described in infants and more rarely in adults [3-8]. The etiology of aorto-ventricular tunnel is uncertain. It probly results from a combination of maldevelopment of the cushions which give rise to the pulmonary and aortic roots, and abnormal separation of these structures which also explains its formation and that of the associated valvar and coronary arterial lesions [3]. Typical presentation

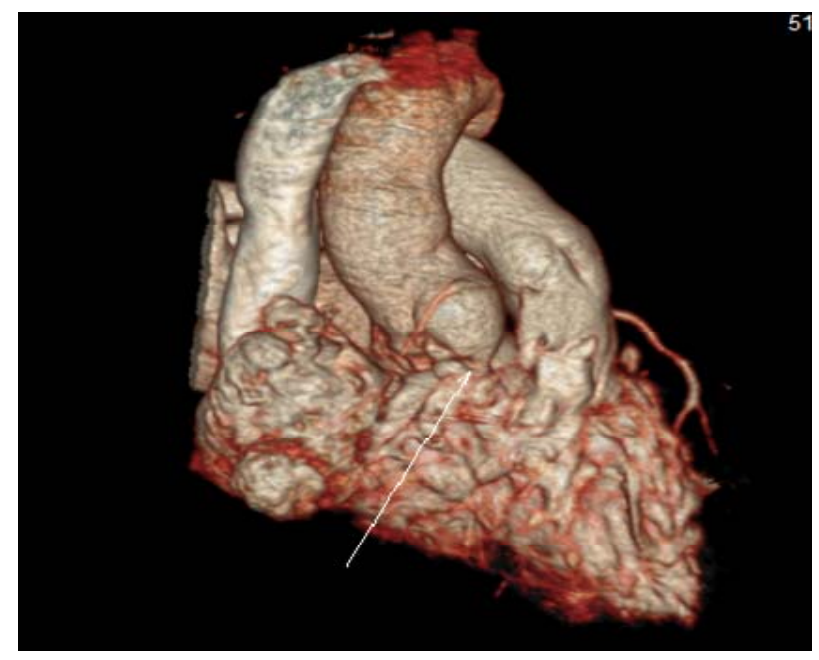

Figure 2. 3D CT angio picture showing aotico-RV tunnel. 


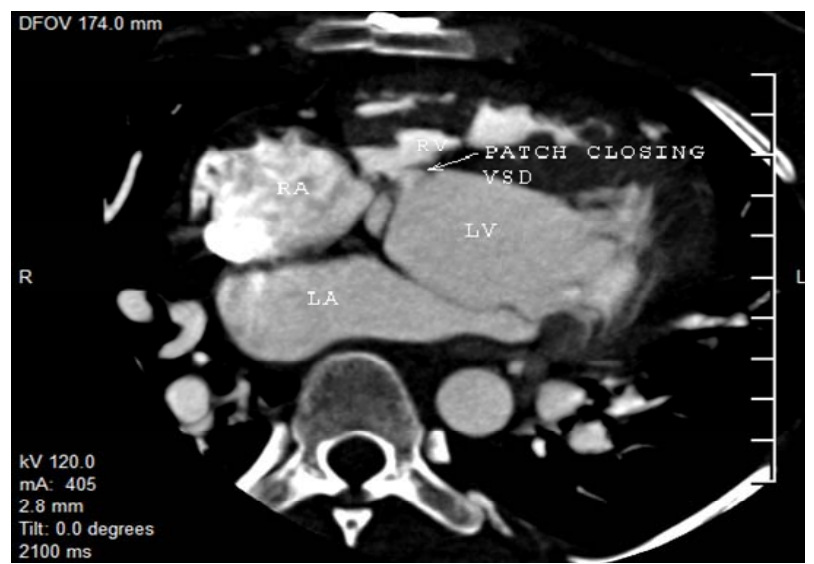

(a)

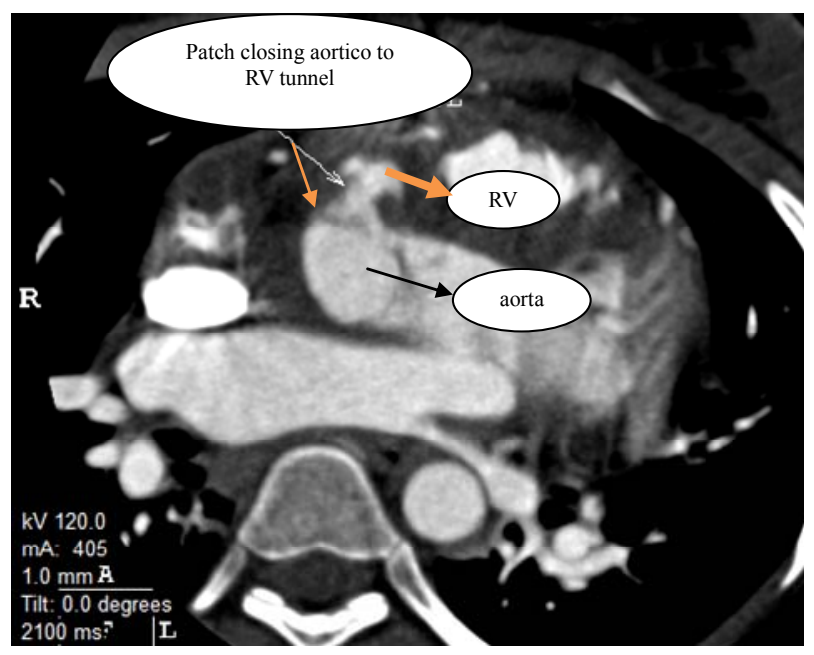

(b)

Figure 3. (a) showing patch closing VSD; (b) showing patch closing aortic-RV tunnel.

has been a critically sick newborn with cyanosis, a "to and fro" murmur, and signs of right heart failure [3,8]. The defect is more common in males and usually is seen in infancy with a to and fro murmur, bounding peripheral pulses, cardiomegaly, and dilatation of the aortic root. The majority of patients have congestive cardiac failure, and a precordial diastolic thrill may be palpable. These findings may also occur with ruptured sinus of valsalva and large coronary artery fistula. Rarity of the condition makes its diagnosis difficult. The correct diagnosis can be made echo-cardiographically and confirmed by cardiac catheterization [3] but the condition may be missed or misdiagnosed as in our case. Our initial diagnosis was ruptured sinus of valsalva with a ventricular septal defect with aortic regurgitation. The diagnosis was made peroperatively and further documented post-operatively with a CT angiography and three dimensional reconstructions [4]. Lin and associates have described this entity in an adult male which was successfully corrected surgically. The diagnosis was preoperatively suspected on the basis of echocardiography with doppler study and it was confirmed by a three-dimensional computed tomographic scan. There may be associated anomaly of coronary artery origin, right ventricular outflow tract, aortic valve abnormality and ventricular septal defect [5-8]. Talwar [5] in their study described an anomalous right coronary artery originating from the distal end of the tunnel. Vargas et al. [6] reported this anomaly in an infant and they found the origin of the aortico-right ventricular tunnel independently from the left coronary ostium and above the sinus of valsalva.

Aortico-right ventricular tunnel should be considered in the differential diagnosis in a critical patient with a "to and fro" murmur, and signs of right heart failure. Before surgical intervention, every effort should be made to diagnose the coronary artery anatomy and surgery should be undertaken soon after the diagnosis is made. The surgical intervention should be individualized based on unique cardiac anomaly of each patient.

\section{REFERENCES}

[1] T. Generali, A. Garatti, A. Biondi, A. Varrica and L. Menicanti, "Aorta to Right Atrial Shunt Due to the Rupture of a Degen-Erative Aneurysm of the Noncoronary Sinus of Valsalva," Journal of Cardiovascular Medicine, Vol. 14, No. 1, 2013, pp. 71-73. doi:10.2459/JCM.0b013e3283528f8c

[2] B. Topi, J. John, A. Agarwal, N. Nerella, V. Shetty, A. Sadiq and J. Shani, "An Uncommon Cause of a Continuous Murmur," Experimental \& Clinical Cardiology, Vol. 17, No. 3, 2012, pp. 148-149.

[3] R. McKay, “Aorto-Ventricular Tunnel," Orphanet Journal of Rare Diseases, Vol. 2, 2007, p. 41. doi:10.1186/1750-1172-2-41

[4] B. S. Lin, X. H. Zhang, Y. Z. Jiang, B. M. Zhang, D. Gao and J. Song, "Diagnosis and Surgical Treatment of Adult Aortico-Right Ventricular Tunnel," The Annals of Thoracic Surgery, Vol. 89, No. 6, 2010, pp. 2024-2026. doi:10.1016/j.athoracsur.2009.10.073

[5] S. Talwar, U. K. Choudhary, S. S. Kothari and B. Airan, "Aortico-Right Ventricular Tunnel," International Journal of Cardiology, Vol. 70, No. 2, 1999, pp. 201-205. doi:10.1016/S0167-5273(99)00059-5

[6] F. J. Vargas, A. Molina, J. C. Martinez, M. E. Ranzini and J. C. Vazquez, "Aortico-Right Ventricular Tunnel," The Annals of Thoracic Surgery, Vol. 66, No. 5, 1998, pp. 1793-1795. doi:10.1016/S0003-4975(98)00927-8

[7] J. A. Van Son, J. Hambsch, P. Schneider and F. W. Mohr, "Repair of Aortico-Right Ventricular Tunnel," European Journal Cardio-Thoracic Surgery, Vol. 14, No. 2, 1998, pp. 214-217. doi:10.1016/S1010-7940(98)00168-7

[8] S. Westaby and N. Archer, "Aortico-Right Ventricular Tunnel," The Annals of Thoracic Surgery, Vol. 53, No. 6, 1992, pp. 1107-1109.

doi:10.1016/0003-4975(92)90401-O 